\title{
Power Allocation by PSO in MAC
}

\author{
Zhang Jingxi \\ School of Electrical and Electronic Engineering, North China Electric Power \\ University, Baoding, Hebei, 071003, China. \\ email: zhjxgeb@sohu.com
}

Keywords: AWGN; MAC; Sigma Mapping; Channel Capacity; PSO; UEP

\begin{abstract}
The capacity of Additive White Gaussian Noise (AWGN) Multiple Access Channel (MAC) can be approached by means of power allocation. The MAC can be regarded as single user multiple level coding and modulation system by applying pulse amplitude modulation to the individual signal and superimposing the weighted signal. Sigma mapping is applied such that the mutual information is as close as possible to the channel capacity. In the design of sigma mapping, every individual signal is weighted by the appropriate power which is optimized by means of Particle Swarm Optimization (PSO). In the respect of approaching MAC capacity and computational complexity, the proposed optimization method is superior to that of Interior Point Method (IPM) and differential evolution. The optimized power can facilitate approaching MAC capacity in practical communication system.
\end{abstract}

\section{Introduction}

Additive White Gaussian Noise (AWGN) Multiple Access Channel (MAC) is widely used in communication systems. The capacity region $\mathrm{C}$ of MAC, i.e., the set of all the combinations of communication rates that are simultaneously achievable with each user guaranteed an arbitrarily small probability of error has been discovered [1]. The method to approach MAC capacity is worth studying because MAC appears naturally in various occasions. In MAC, the individual bits of different users of most digital audio and video transmission systems exhibit different bit error sensitivities.

Channel coding with unequal error protection (UEP) is generally applied. However, some transmission systems, e.g., DECT and Bluetooth, include only weak or no channel coding for some reasons. A novel concept named modulation with unequal power allocation (MUPA) is proposed for this situation. It can achieve UEP by periodically allocating unequal transmission power to information bits of different users. MUPA is carried out in the context of BPSK modulation. UEP can be realized by bit-wise control of the BPSK modulation amplitudes. The different amplitude levels are obtained by systematically trying out a number of energy distributions [2]. Taking the mean square error as optimization criterion in the parameter domain, an analytical approach was presented with numerical optimization for arbitrary bit mappings [3].

A one-layer coding/shaping scheme of a perfectly cooperated multiple-access system was investigated in [4]. At the transmitter, binary data are encoded by either single-level or multilevel codes. The coded bits are then fed into a signal mapping, which accepts at any time input multiple binary digits and outputs an amplitude signal. In other words, the input are independently mapped into 2-PAM signals (possibly having different amplitudes) and superimposed to form the output. Therefore assigning different power to different bits of individual user is of great importance. Ma [4] used Gallager mapping [5] to approach channel capacity. First the signal mapping is applied such that the mutual information is as close as possible to the channel capacity, and then an outer binary code is designed to approach the mutual information.

With an effort to approach MAC capacity, Wang [6] used IPM and obtained power profile in cases of 4, 8, 16 users. The disadvantage of IPM method is its high computation cost and no guarantee of convergence.

This paper aims at approaching the MAC capacity by power allocation in AWGN MAC.Power 
allocation is explored in the context of 2-PAM modulation. By proper designing sigma mapping [5] with optimized power to different users MAC capacity can be approached. Power optimization is carried out by Particle Swarm Optimization (PSO) which is a stochastic optimization technique and has been widely used to solve a range of optimization problems and can be likened to the behavior of a flock of birds or the sociological behavior of a group of people [7]. Channel coding is not considered for the moment. At a given SNR, power profile was searched by PSO to approach the limit of MAC capacity region. The sum power constraint is assumed for the MAC, meaning that there are no constraints on the powers of individual transmitters, but rather a global constraint on the total power across all users and channels.

The paper was arranged as follows: Section II introduces the system model of signal mapping. Section III gives an overview of MAC channel capacity. Section IV elaborates the method used in searching the power profile. Section V performs a comparison of mutual information obtained with target power profile and with the searched profile. Section VI concludes the paper..

\section{System Model}

In MAC situation $\mathrm{K}$ users transmit their respective signal a common receiver simultaneously. The system model can be illustrated in Fig.1. Suppose user $k$ sends data $d k, k \in\{0,1,2, K-1\}$ at some time instance, where $\mathrm{k}$ has generalized meaning, it can be $\mathrm{k}$ parallel data from one user. The encoders vary with specific requirement of different "users". After coding $\{\mathrm{c} 0, \mathrm{c} 1, \ldots, \mathrm{ck}-1\}$ will be generated. Then the mapping operation is carried out on the coded data, generating $\mathrm{x}$. In this way the MAC channel resembles single user one layer coding/shaping scheme. AWGN noise is added to the mapped signal $\mathrm{x}$, forming channel output $\mathrm{y}$. The BPSK modulation is considered without coding for the sake of simplicity. By proper assigning power between different users, we manage to approach MAC channel capacity.

The ideal base band discrete-time model for the uplink AWGN channel with $\mathrm{K}$ users is characterized by

$$
Y_{t}=X_{t}+W_{t}
$$

where $\mathrm{Xt} \in \mathrm{R}$ and $\mathrm{Yt} \in \mathrm{R}$ are the channel input and output at time t, respectively.

$$
x=\phi_{\Sigma}(v)=\sum_{i=0}^{k-1} \alpha_{i}\left(1-2 v^{(i)}\right)
$$

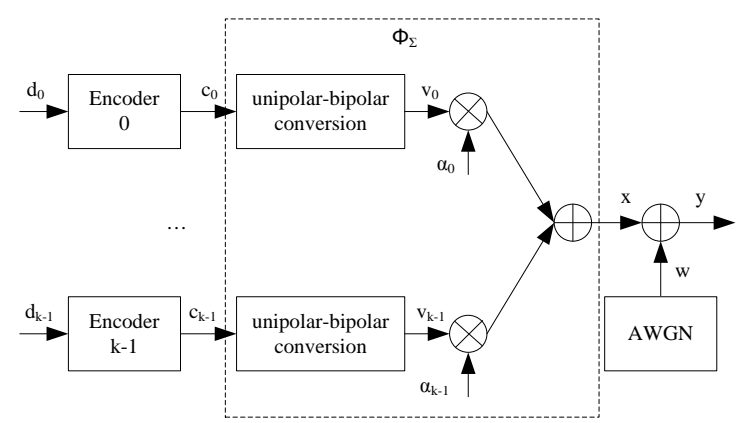

Fig. 1 coding and mapping

The additive noise sequence $\mathrm{W}=\left(\mathrm{W}_{1}, \ldots, \mathrm{W}_{\mathrm{t}}\right)$ is assumed to be an i.i.d. sequence with mean 0 and variance $\sigma^{2}$, denoted by

$$
W_{t} \sim N\left(0, \sigma^{2}\right)
$$

User $\mathrm{k}$ has an average power constraint of $\mathrm{P}_{\mathrm{k}}$ joules/symbol (with $\mathrm{k}=0,1, \mathrm{~K}-1$ ), i.e.,

$$
E\left(X_{t}^{2}\right) \leq E_{s}
$$

where $\mathrm{E}(\cdot)$ is the statistical expectation and $E_{s}$ denotes the energy per symbol. 


\section{MAC Channel Capacity}

The MAC capacity is a capacity region $C$ : this is the set of all pairs $\left(\mathrm{R}_{0}, \mathrm{R}_{1}, \ldots, \mathrm{R}_{\mathrm{K}-1}\right)$ such that $\mathrm{K}$ users can reliably communicate at rate $\mathrm{R}_{0}, \mathrm{R}_{1}$ and $\mathrm{R}_{\mathrm{K}-1}$, respectively[8]. Since $\mathrm{K}$ users share the same bandwidth, there is naturally a tradeoff between the reliable communication rates of the users. The sum capacity:

$$
C_{\text {sum }}:=\max _{\left(R_{0}, R_{1}, \ldots, R_{K-1}\right) \in C}\left(\sum_{i=0}^{K-1} R_{i}\right)
$$

is the maximum total throughput that can be achieved.

The K-user capacity region is described by $2^{\mathrm{K}}-1$ constraints, one for each possible non-empty subset $\mathrm{S}$ of users:

$$
\sum_{k \in S} R_{i}<\frac{1}{2} \log _{2}\left(1+\frac{\sum_{k \in S} P_{k}}{\sigma^{2}}\right) \quad S \subset\{0,1, \ldots, K-1\}
$$

(5) and (6) show that the capacity region $C$ is a closed convex polyhedron. The number of vertices whose coordinates are all positive is exactly $n$ !. The meaning of (6) is that the sum rate of any subset $\mathrm{S}$ of users in this system is less than the rate obtained as if one user had the entire received power of subset $S$ and with no other users in the system, denoted by

$$
C=\frac{1}{2} \log _{2}\left(1+\frac{\sum_{k=0}^{K-1} P_{k}}{\sigma^{2}}\right) \mathrm{S} \subset\{0,1, \ldots, \mathrm{K}-1\}
$$

Thus, the optimal power allocation problem should be thought of as how to partition the total power across the time/frequency degrees of freedom and how to share the resource across the users.

\section{Problem Formulation and Solution}

The target function is mutual information. The objective is to make mutual information as large as possible by searching optimized power profile at given SNR. In mathematical form,

$$
\max \left(I_{\phi}(S N R)\right)=\max \left(E\left(\log _{2} \frac{P_{Y / X}(y / x)}{P_{Y}(y)}\right)\right)
$$

subject to the following constraint

$$
\left\{\begin{array}{l}
P_{i}=\alpha_{i}^{2} \\
S N R_{i}=\frac{P_{i}}{\sigma^{2}} \\
S N R=\sum_{i=1}^{k} S N R_{i} \\
x=\sum_{i=0}^{k-1} \alpha_{i}\left(1-2 v^{(i)}\right)
\end{array}\right.
$$

Without loss of generality, assume $\sigma^{2}=1$. In this way, the SNR changes with $\mathrm{P}_{\mathrm{i}}$. Let the user number be $\mathrm{K}$ which is a positive integer and $\mathrm{V}=\left(\mathrm{V}^{(0)}, \ldots, \mathrm{V}^{(\mathrm{K}-1)}\right)$ be a binary i.i.d. sequence from $\mathrm{K}$ users with realizations $\mathrm{v} \in \mathrm{F}_{2}{ }^{\mathrm{k}}$. Denote $\mathrm{Y}$ the observation of $\mathrm{X}$ at the output of the AWGN channel. Then $\mathrm{V} \rightarrow \mathrm{X} \rightarrow \mathrm{Y}$ forms a Markov chain. The mutual information $\mathrm{I}_{\Phi}$ of the given signal mapping $\Phi$ in (2) is defined as the mutual information $\mathrm{I}(\mathrm{V} ; \mathrm{Y})$.

$$
I_{\phi}=I(V ; Y)=I(X ; Y)=E\left(\log _{2} \frac{P_{Y / X}(y / x)}{P_{Y}(y)}\right)
$$

where

$$
P_{Y / X}(y / x)=\frac{1}{\sqrt{2 \pi}} \exp \left(-(y-x)^{2} / 2\right)
$$

is the channel transition probability density function (pdf) and 


$$
P_{Y}(y)=\sum_{x} P_{X}(x) P_{Y / X}(y / x)
$$

The threshold SNR requirement for decoding/ demodulation is not considered here specifically since error-free transmission can be guaranteed by powerful variable rate error-correcting code with rate determined by power[9]. PSO algorithm is inspired by social behavior of bird flocking or fish schooling. In PSO, each single solution is a "bird" which is called a "particle" in the search space. All of particles have cost values which are evaluated by the cost function to be optimized, and have velocities which direct the flying of the particles. The particles fly through the problem space by following the current optimum particles. Every particle in the solution space will search individual optimization result in the solution space and label it with $p_{\text {best }}$, meaning the position vector of the best solution this particle has achieved so far.; then compare all the individual optimization result to get the global Optimization result labeled $f\left(g_{\text {best }}\right)$, where $g_{\text {best }}$ is the particle which obtains the global optimization result. Next "acceleration" process is conducted in the algorithm, i.e. all the particle "flying" to the region specified by $p_{\text {best }}$ and $g_{\text {best }}$. Random accelerated factor is introduced in "acceleration”.

In this optimization process, PSO algorithm starts from a group of solution (particles) with population size $\mathrm{N}$. The initial values of the elements of each solution $\left(p_{1}, p_{2}, \ldots, p_{m}\right)$ are randomly generated subject to the constraints(9). And then iteratively searches the optima by updating generations. When the position and velocity of each updated particle is greater than their upper bound, they are limited to these maximum values. The power optimization process can be briefly described as follows.

1) Initialization: generation of power randomly subject to the constraints (9).

2) Adjust the position of each particle based on (14).

3) Compare the cost function $f(p)$ (here is mean mutual information) of the particle $\mathrm{p}$ and individual optimization value $f\left(p_{\text {best }}\right)$; if $f(p)>f\left(p_{\text {best }}\right)$, update is made, i.e. $p_{\text {best }}=p$; else $p_{\text {best }}$ keeps unchanged.

4) Compare the current global optimization result $f(g)$ and the optimization result $f\left(g_{\text {best }}\right)$; if $f(g)>f\left(g_{\text {best }}\right)$, update is made, i.e. $g_{\text {best }}=g$ and $f\left(g_{\text {best }}\right)=f(g)$; else $f\left(g_{\text {best }}\right)$ keeps unchanged.

5) Modify the velocity of particle:

For each generation the velocity of each particle is updated as

$$
v_{i d}=w \cdot v_{i d}+c_{1} \cdot \operatorname{rand}_{1} \cdot\left(p_{i d}-x_{i d}\right)+c_{2} \cdot \operatorname{rand}_{2} \cdot\left(p_{g d}-x_{i d}\right)
$$

where $w$ is an inertial parameter, $\quad \mathrm{V}_{\text {id }}$ is the velocity in the d-th dimension of the $\mathrm{i}$-th particle, $c_{1}$ and $c_{2}$ are weighting coefficients which is constant accelerate factor, rand $_{1}$ and rand $_{2}$ and are random numbers uniformly distributed over $(0,1), p_{i d}$ is $p_{\text {best }}$ position of the d-th dimension of the i-th particle, $x_{i d}$ is the position in the d-th dimension of the i-th particle; $p_{g d}$ is the position of d-th dimension of the particle $g_{\text {best }}$. And the position of the solution was updated as

$$
x_{i d}=x_{i d}+v_{i d}
$$

The position and speed of each particle are limited to their maximum range.

6) Stopping criterion: if the maximum iteration numbers is reached, then the iteration stops and record the optimization result; else return to 2). 


\section{Comparison of Mutual Information}

This part gives the computational result of AWGN MAC channel capacity limit, the attainable AWGN MAC channel capacity by equal and unequal power allocation between different users, and the optimized power profiles obtained by the aforementioned PSO method.

The theoretical channel capacity of AWGN MAC is calculated based on (7) and it is drawn in Fig. 2 in a black solid line with a point. In PSO process, $\mathrm{c}_{1}=2, \mathrm{c}_{2}=2, \mathrm{w}=0.5$. The attainable AWGN MAC channel capacity by equal power allocation between 2, 4 and 8 users are shown in Fig.2 in black solid line with a left triangle, plus and start, respectively. The attainable AWGN MAC channel capacity by unequal power allocation is also shown in Fig.2 with dotted line. The SNR ranges from 0 to $30 \mathrm{~dB}$.

It can be seen from fig.2 that the attainable AWGN MAC capacity limits increases with "users" in both equal power and unequal power allocation cases. It can also be seen that the asymptotic attainable AWGN MAC capacity limits with unequal power allocation is better than those of equal power allocation between "users" in every case.

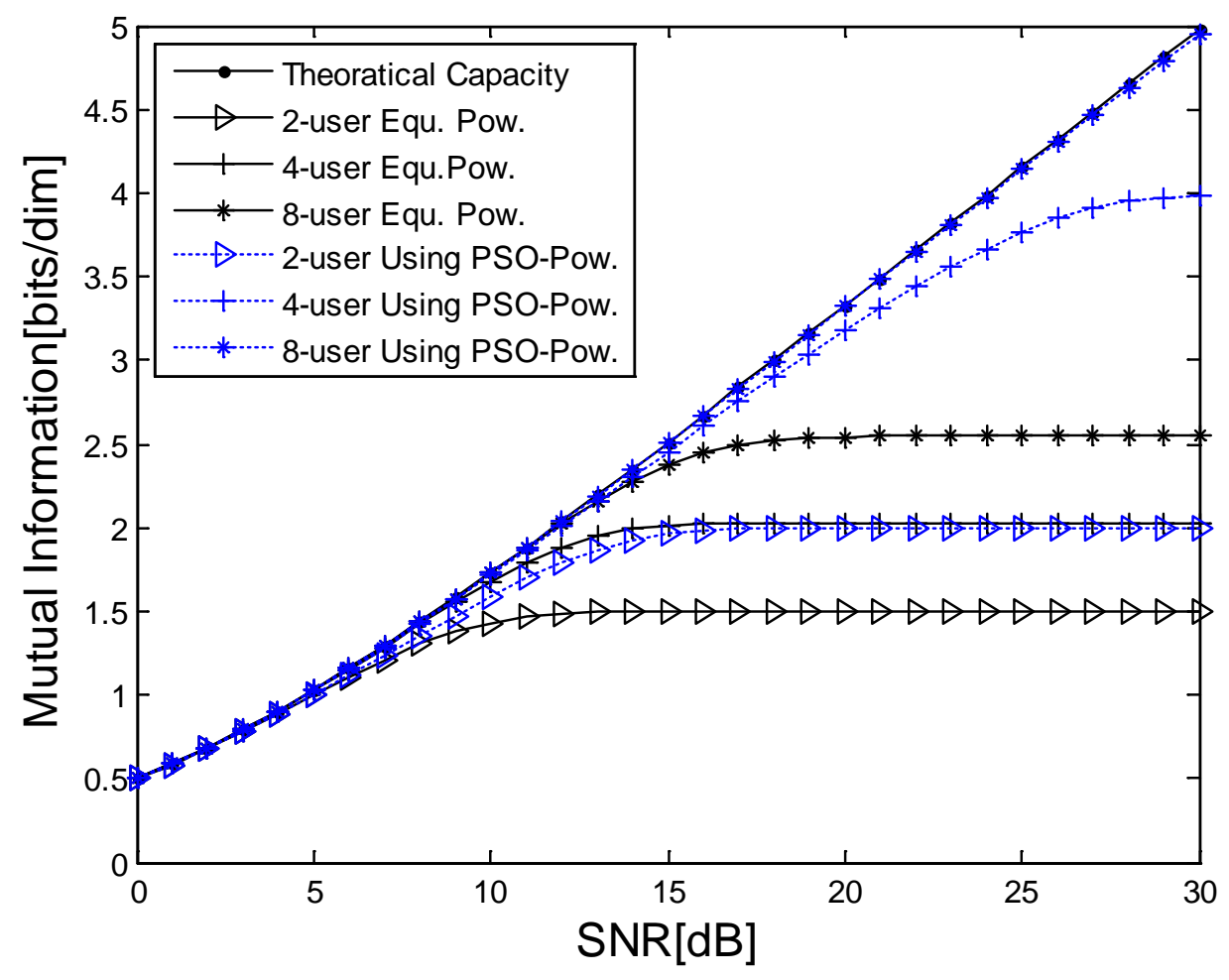

Fig.2 Comparison of Mutual information with Different Users and Power

It can be seen from fig. 2 that the attainable AWGN MAC capacity limits increases with "users" in both equal power and unequal power allocation cases, and the asymptotic attainable AWGN MAC capacity limits with unequal power allocation is better than those of equal power allocation between "users" in every case. Tab. 1 shows the optimized power profile for 4 and 8 user cases with SNR from 0 to $30 \mathrm{~dB}$. Fig.3 gives the comparison of mutual Information obtained between optimized power by PSO method and power by IPM method [6]. The target power profile is in listed in Tab.2. 
Tab.1 Optimized Power Profile for 4 and 8 Users

\begin{tabular}{|c|l|l|l|l|}
\hline \multicolumn{1}{|c|}{$\alpha_{\mathrm{i} R}$} & $0 \mathrm{~dB}$ & \multicolumn{1}{|c|}{$10 \mathrm{~dB}$} & \multicolumn{1}{|c|}{$20 \mathrm{~dB}$} & \multicolumn{1}{|c|}{$30 \mathrm{~dB}$} \\
\hline \multirow{3}{*}{4} & 0.490681 & 0.978280 & 1.649732 & 2.792472 \\
users & 0.496755 & 1.428347 & 3.472464 & 6.795254 \\
& 0.505504 & 1.688692 & 6.046849 & 13.505074 \\
& 0.506886 & 2.037428 & 6.975385 & 27.634030 \\
\hline \multirow{3}{*}{8} & 0.274759 & 0.772507 & 1.655950 & 2.927646 \\
& 0.292916 & 0.872777 & 2.134597 & 4.797900 \\
users & 0.307666 & 1.145442 & 2.736636 & 5.686886 \\
& 0.338407 & 1.148542 & 3.259478 & 6.572753 \\
& 0.344180 & 1.198817 & 3.583052 & 11.767484 \\
& 0.388857 & 1.214307 & 3.858281 & 13.326516 \\
& 0.406935 & 1.228409 & 4.542541 & 15.710638 \\
& 0.440754 & 1.60798 & 5.121395 & 18.165172 \\
\hline
\end{tabular}

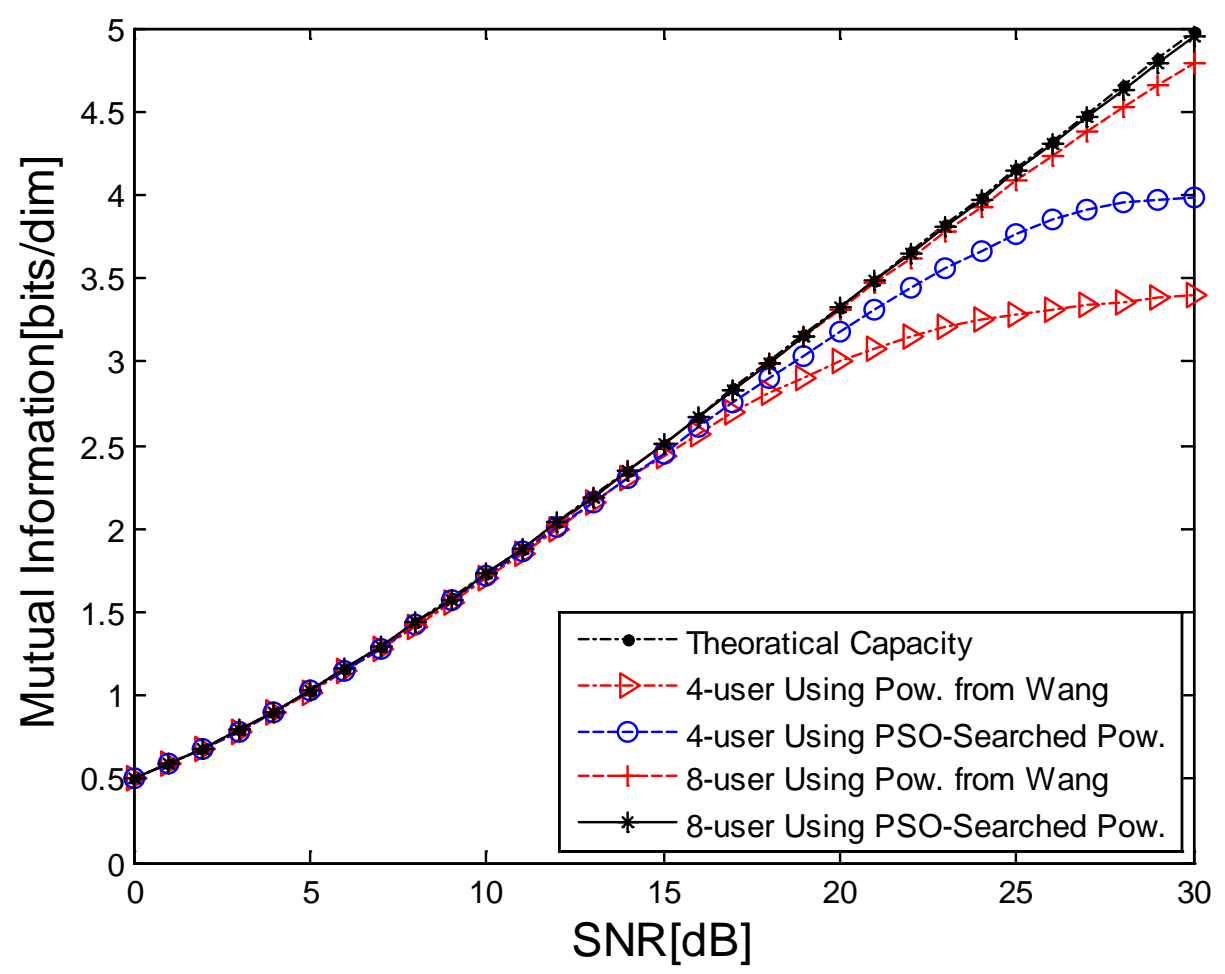

Fig.3 Comparison of Mutual Information obtained by using PSO-Searched Power profile and Target Power Profile from IPM

It can be seen from Fig.3 that the attainable AWGN MAC capacity limits with obtained power profile by PSO method is better than that of target power profile in both 4 and 8 user cases, and the performance gain is obvious when SNR is high. The result shows that the searched power profile is superior to that obtained by IPM method, especially in the case of 4 users.

Tab.2 Target Power Profile

\begin{tabular}{|c|c|}
\hline $\mathrm{K}$ & Relative power levels $\alpha_{\mathrm{i}}{ }^{2}(\mathrm{~dB})$ \\
\hline 4 & $0,0.0021,5.4647,7.5530$ \\
\hline 8 & $0,0.0004,0.0021,0.0097,4.0177,5.7518,6.8848,7.7535$ \\
\hline
\end{tabular}

It should be noted that lowest value of Wang's power profiles is normalized, and Wang's power profile is obtained by confining searching within the feasible region based on a barrier function. Meanwhile we must note that the optimized power profile is dependent on SNR, when SNR 
changes, the optimized power profile should be changed to approach channel capacity.

In terms of approaching attainable AWGN MAC channel capacity, the obtained power profile by PSO method is superior to that of target power profile. And how will this power profile be utilized in MAC to get the desirable concrete result is the next target.

It can be seen from Fig.2 and Fig.3 that more users should be used to approach AWGN MAC channel capacity in both equal and unequal power allocation cases, power optimization should be carried out in MAC cases, and the performance gain reduces with increasing user. .

\section{Conclusion}

MAC is treated as single user multiple level coding and modulation system. Sigma mapping is used in multi-level code and the transmitted signal can be obtained by weighting the modulated signal and summing them. PSO is used to optimize the power of different users. The criterion is to maximize the mutual information between different user's transmitted signal and the output of AWGN MAC subject to the SNR restraint. Optimized power profiles for different users under different SNR are obtained. The method can approach MAC capacity with reduced computational complexity. And the optimized power can facilitate approaching MAC capacity in practical communication system. The optimization of power profile is considered in the case of AWGN MAC in this paper, and the method can be easily extended to fading MAC, which needs further study.

\section{Acknowledgement}

The paper is supported by "the Fundamental Research Funds for the Central Universities(9161113003)”.

\section{References}

[1] T. M. Cover and J. A. Thomas, Elements of Information Theory. New York: Wiley, 1991.

[2] N. G“ortz and E. Bresch, "Source-Adaptive Power Allocation for Digital Modulation,” IEEE Commun. Lett., vol. 7, pp. 569-571, Dec. 2003.

[3]Thomas Br"uggen and Peter Vary. "Unequal Error Protection by Modulation with Unequal Power Allocation,” IEEE Commun. Lett., VOL. 9, NO. 6,pp.484-486 Jun. 2005.

[4] X. Ma and L. Ping, “Coded modulation using superimposed binary codes,” IEEE Trans. Inform. Theory, vol. 50, pp. 3331-3343, Dec. 2004.

[5]R. G. Gallager, Information Theory and Reliable Communication. New York: Wiley, 1968.

[6]Peng Wang; Li Ping; Lihai Liu, "Power Allocation for Multiple Access Systems with Practical Coding and Iterative Multi-User Detection," in Proc. IEEE International Conference on Communications, Istanbul, Turkey, Jun. 2006,Vol.11, pp.4971-4976.

[7] Frans van den Bergh and Andries P. Engelbrecht, "A Cooperative Approach to Particle Swarm Optimization.”, IEEE Trans on evolutionary computation, vol.8, no. 3, pp225-239 Jun. 2004.

[8] D. Tse and P. Viswanath, Fundamentals of Wireless Comrnunication, Cambridge University Press, 2005.

[9] S. Vishwanath, S. A. Jafar, and A. I. Goldsmith. "Optimum power and rate allocation strategies for multiple access fading channels,” in Proc. Spring IEEE VTC. vol. 4, Rhodes, Greece, May 2001, pp. 2888-2892. 